\title{
RIPK3在磨损颗粒激活巨噬细胞PRRs/NLRP3 信号通路引起人工关节无菌性松动中的作用机制
}

\author{
彭芒 ${ }^{\dagger}$, 傅媛 ${ }^{\dagger}$, 邱俊雄, 李仕勋, 李长川, 邨满源, 房桂涁, 林思鹏, 丁悦 ${ }^{*}$ \\ 中山大学孙逸仙纪念医院骨外科, 广州 510120 \\ $\dagger$ 同等贡献 \\ *联系人, E-mail: dingyue@mail.sysu.edu.cn
}

收稿日期：2020-01-15; 接受日期：2020-03-11; 网络版发表日期：2020-06-04

国家自然科学基金(批准号: 81672186)资助

\begin{abstract}
摘要人工关节置换术是治疗各种终末期骨关节疾病及部分骨肿瘤疾病的最有效手段, 但人工关节假体的使用 寿命有限. 无菌性松动是限制人工关节假体远期使用寿命的主要原因, 其中磨损颗粒激活巨噬细胞产生促炎因子 导致的假体周围骨溶解被认为是无菌性松动发生的最重要因素。磨损颗粒能激活巨噬细胞的模式识别受体(pattern recognition receptors, PRRs)从而产生TNF- $\alpha$ 和IL-1 $\beta$ 等促炎因子, 诱发假体周围骨溶解. 本文在之前研究的基 础上发现, 在抑制RIPK3(receptor-interacting protein kinase 3)基因的小鼠巨噬细胞中, 磨损颗粒诱导产生的促炎因 子表达量显著下调，且NLRP3炎性小体组装受限。同时发现，注射靶向抑制RIPK3慢病毒的小鼠彩骨溶解效应减 弱. 本研究表明, RIPK3 在磨损颗粒激活巨噬细胞PRRs/NLRP3信号通路引起炎症反应中起正性调控作用.
\end{abstract}

关键词 RIPK3, NLRP3, 人工关节置换, 假体无菌性松动, 巨噬细胞

人工关节置换术是治疗各种终末期骨关节疾病及 部分骨肿瘤疾病的最有效手段 ${ }^{[1]}$. 随着中国老龄化社 会的到来以及中国经济水平的飞快增长，中国目前每 年有超过 50 万台人工关节假体置换手术，但人工关节 假体的使用寿命有限, 通常为 $10 \sim 15$ 年 ${ }^{[2]}$. 最新一项研 究通过对23269例髋关节翻修患者进行回顾性调查发 现, 无菌性松动在所有翻修原因中所占比例最高, 超过 $50 \%{ }^{[3]}$. 然而目前尚无防治人工关节无菌性松动的有 效方法. 翻修手术难度大、创伤多、费用高、术后并 发症多，且会进一步增加再次翻修的风险，造成恶性
循环. 如何防治人工关节无菌性松动是目前骨科界最 大的挑战和难题.

人工关节远期无菌性松动发生机制包括生物因 素、假体微动、应力遮挡、个体遗传差异等, 其中磨 损颗粒诱导的假体周围骨溶解是最关键的原因 ${ }^{[4]}$. 本 课题组的前期研究表明, 假体来源的磨损颗粒(包括金 属钛颗粒、超高分子聚乙烯、骨水泥分子等)可激活 巨噬细胞NF-kB信号通路产生促炎因子. 磨损颗粒的 直径、形态以及浓度均可以影响促炎因子的产生 ${ }^{[5]}$.

巨噬细胞促炎因子的产生与巨噬细胞表面的模式

引用格式: 彭茫, 傅媛, 邱俊雄, 等. RIPK3在磨损颗粒激活巨噬细胞PRRs/NLRP3信号通路引起人工关节无菌性松动中的作用机制. 中国科学: 生命科学, 2020, 50: 1121-1131

Peng P, Fu Y, Qiu J X, et al. Mechanism of RIPK3 in activation of PRRs/NLRP3 signaling pathway in macrophages induced by wear particles in aseptic joint loosening (in Chinese). Sci Sin Vitae, 2020, 50: 1121-1131, doi: 10.1360/SSV-2020-0016 
识别受体(pattern recognition receptors, PRRs)密切相 关 ${ }^{[6]}$. PRRs是表达在固有免疫细胞表面的特定识别分 子，是固有免疫的重要组成部分. 其中TLRs(Toll-like receptors)在固有免疫中发挥重要作用，TLRs是一 种 I 型跨膜蛋白, 分布在固有免疫细胞的表面 ${ }^{[7]}$. 当有 外来刺激物接触巨噬细胞时, TLRs (主要为TLR4和 TLR2)能识别外来刺激物的PAMPs/DAMPs(damageassociated molecular patterns), 在PAMPs诱导机体产生 炎症反应的过程中发挥始动作用 ${ }^{[8,9]}$. 近几年发现, TLRs信号通路下游中的NLRP3炎性小体参与了机体 相关的多种炎症反应. NLRP3是NLRs(NOD-like receptors)家族中的重要成员，广泛表达于包括巨噬细胞在 内的各种髓系细胞 ${ }^{[10]}$. NLRP3蛋白可被多种机体疾病 相关的细菌、病毒、尿酸结晶等PAMPs/DAMPs激活, 其激活过程与PRRs(如TLRs, NOD2)以及TNF- $\alpha$ (tumor necrosis factor $\alpha$ )等促炎因子均密切相关 ${ }^{[11]}$. 研究表明, 细胞表面的TLRs识别PAMPs/DAMPs后可激活其下游 的NF- $\kappa$ B信号通路, 进而上调NLRP3蛋白的表达 ${ }^{[12]}$. 同 时被激活后释放的 TNF- $\alpha$ 及IL- $1 \beta$ 等促炎因子可正反馈 上调NLRP3蛋白的表达 ${ }^{[13]}$. 因此NLRP3与机体各种炎 症反应息息相关. NLRP3蛋白被激活后, 可与适配蛋白 ASC(apoptosis-associated speck-like protein containing CARD) 以及pro-Caspase-1共同组装成NLRP3炎性小 体, 这是NLRP3炎性小体组装形成的主要途径 ${ }^{[14]}$. NLRP3炎性小体可将pro-Caspase-1活化为活性Caspase-1，后者可将促炎因子前体如pro-IL-1 $\beta$, pro-IL-18 切割为成熟的、具有活性的促炎因子，如IL- $1 \beta$ 和IL18等, 从而发挥促炎作用.

RIPK(receptor-interacting protein kinase, 也称receptor-interacting protein, RIP)家族是一类丝氨酸-苏 氨酸蛋白激酶, RIPK 3 是人类RIPK家族RIPK $1 \sim R I P K 5$ 五个成员之一. RIPK3蛋白含有 518 个氨基酸: 其氮端 为 RIPK家族中保守的激酶结构域，与RIPK3活性相 关; 碳端为RHIM(RIP homotypic interaction motif)结 构域, 可与其他蛋白的RHIM结构域相互作用 ${ }^{[15]}$. 通常 认为, RIPK3 与调亡反应密切相关, 甚至在部分细胞凋 亡过程中起分子开关的作用. 但近期越来越多的研究 表明，RIPK3在炎症通路上也发挥关键作用. Wang等 人 $^{[16]}$ 在Nature Immunology 上的研究表明, 巨噬细胞的 RIPK3 在NLRP3炎性小体的组装激活中发挥关键作 用. 此外, RIPK3与巨噬细胞TLRs通路也密切相关 ${ }^{[17]}$.
但在人工关节无菌性松动的领域中，目前仍未有任何 报道针对RIPK3 在磨损颗粒激活巨噬细胞引起人工关 节无菌性松动过程中的作用机制进行探讨. 对此，本 课题组在体内、体外实验中明确了RIPK3 在磨损颗粒 激活巨噬细胞引起人工关节无菌性松动中的作用及 机制, 为人工关节无菌性松动提供潜在治疗靶点.

\section{1 材料与方法}

\section{1 髋关节滑膜标本来源及取材方法}

髋关节标本来源：所有标本均取自中山大学孙逸 仙纪念医院骨科和佛山市第一人民医院骨科. 收集时 间为 20 个月(2015年3月 2016年10月). 收集标准为: (i) 排除合并有自身免疫性疾病、感染、免疫缺陷型 疾病的患者病例; (ii) 符合股骨头坏死ARCO(The Association Research Circulation Osseuse) IV 期标准且为 初次人工全髋关节置换术, 纳入股骨头坏死组; (iii) 符 合人工髋关节假体松动标准且为第二次人工关节置换 术的患者, 纳入无菌性松动组. 符合( i )( ii)两条标准 或者( i )(iii)两条标准可收集入组. 无菌性松动组选取 患者假体周围组织, 其他组选取患者髃关节滑膜组织, 使用 $10 \%$ 福尔马林固定液固定保存. 最后收集标本分 为两组: 股骨头坏死组: 8例(男性4例, 女性4例), 无菌 性松动组: 7例(男性3例, 女性4例), 平均取材时间在初 次置换术后 $(15.090 \pm 3.032)$ 年.

\section{2 免疫组化染色及染色评分}

连续切片后使用二甲苯脱蜡, 再使用 $3 \%$ 过氧化氢 封闭过氧化物酶, 依次利用从高至低的各浓度酒精各 浸泡 3 min. 高压修复暴露抗原, phosphate buffered saline $(\mathrm{PBS})$ 浸洗 3 次后加入稀释一抗， $4^{\circ} \mathrm{C}$ 孵育 $12 \mathrm{~h}$. PBS浸洗3次后使用DAB(diaminobenzidine)进行显色, 再使用苏木素进行染核, 放置于 $65^{\circ} \mathrm{C}$ 烘片箱内 $1 \mathrm{~h}$ 后封 片. 采用Bresaliers半定量评分法 ${ }^{[18]}$ 评价免疫染色玻片 结果: 两名科研人员采用双盲法在切片中随机选取 10 个视野，根据组织中目的蛋白的染色强度分为四个等 级, 计算每种染色强度的细胞在该视野中的细胞总数, 计算出各个视野中不同染色强度分值与其在该视野 总细胞数目所占比例的的乘积之和. 染色强度得分 $(\mathrm{IS})=0 \times \mathrm{F} 0+1 \times \mathrm{F} 1+2 \times \mathrm{F} 2+3 \times \mathrm{F} 3$. 最终取平均值得到最 后染色评分结果. 


\section{3 磨损钛颗粒的制备材料和方法}

将钛颗粒(ALFA AESAR公司，德国)充分溶解于 无菌纯水中. 准备过滤装置2台(MILLIPORE公司, 美 国): 装置1安装 $1.2 \mu \mathrm{m}$ 滤膜; 装置2安装 $0.2 \mu \mathrm{m}$ 滤膜. 将 磨损钛颗粒先后经过两台过滤装置过滤, 得到直径级 别在0.2 1.2 $\mu \mathrm{m}$ 的钛金属颗粒. 洗脱滤膜上的金属钛 颗粒, $65^{\circ} \mathrm{C}$ 烘干 $1 \mathrm{~h}$, 环氧乙烷气体消毒. 以内毒素试剂 盒确认钛金属颗粒悬液中的内毒素已经完全清除. 按 照 $0.0091 \mathrm{~g} / \mathrm{mL}$ 的溶度配置钛颗粒溶液. 钛颗粒溶液于 $-20^{\circ} \mathrm{C}$ 环境下保存.

\section{4 巨噬细胞J774A.1的刺激方法}

将巨噬细胞J774A.1(ATCC, 美国)进行分组种板, $24 \mathrm{~h}$ 观察巨噬细胞活度和显微镜下细胞种植密度(细 胞种植密度 $\geq 70 \%$ ), 按照以下方法进行刺激.

刺激方法 1 : 使用 $200 \mu \mathrm{L}$ 钛颗粒溶液刺激J774A.1 巨噬细胞，分别提取刺激后 $0 \mathrm{~h}, 1 \mathrm{~h}, 2 \mathrm{~h}, 4 \mathrm{~h}, 6 \mathrm{~h}$ 的 RNA样本.

刺激方法2: 将巨噬细胞J774A.1分为 3 组种板, 按 以下方法进行刺激: A组 $2 \mu \mathrm{L} \mathrm{LPS}(100 \mathrm{ng} / \mathrm{mL})+200 \mu \mathrm{L}$ PBS; B组 $2 \mu \mathrm{L}$ LPS(100 ng/mL) $+200 \mu \mathrm{L}$ 钛颗粒溶液; C 组 $2 \mu \mathrm{L} \mathrm{LPS}(100 \mathrm{ng} / \mathrm{mL})+200 \mu \mathrm{L} \operatorname{ATP}(10 \mathrm{mg} / \mathrm{mL})$. 分别 提取3组刺激后细胞的蛋白.

\section{5 靶向敲低 RIPK3 基因的siRNA构建、慢病毒 构建及转染}

在PubMed上查询目的基因 RIPK3 的基因编号及 相关基因序列. 将该序列交由上海吉玛公司设计合成 small interfering RNA (siRNA). 合成序列如表1所示. 配置转染体系： $400 \mu \mathrm{L}$ 无血清Opti-MEM培养基, 24 pmol siRNA, $4 \mu \mathrm{L}$ 的RNAiMAX. 充分混匀后孵育 $15 \mathrm{~min} .2 \mathrm{~h}$ 后将转染体系加入六孔板中 $(400 \mu \mathrm{L} /$ 孔), 效 率检测后选取敲低效率最高的RIPK3-1128及RIPK3493序列进行构建重组慢病毒. 将上述序列合成包装成 有 short hairpin RNA(shRNA)和green fluorescent protein (GFP)的慢病毒(滴度: $8 \times 10^{8} \mathrm{TU} / \mathrm{mL}$ ). 六孔板每孔种植 $4 \times 10^{5}$ 个巨噬细胞, $24 \mathrm{~h}$ 后使用无血清Opti-MEM培养 基换液培养. 构建慢病毒转染体系: $200 \mu \mathrm{L}$ 慢病毒 +4 $\mu \mathrm{g}$ polybrene混匀后孵育 $10 \mathrm{~min} .2 \mathrm{~h}$ 后将转染体系加入 六孔板中 $(200 \mu \mathrm{L}$ 孔), 消化细胞, 离心洗涤后送流式细
表 1 RIPK3-siRNA合成序列

Table 1 Synthetic sequences of RIPK3-siRNA

\begin{tabular}{cc}
\hline 基因编号 & 基因片段 $\left(5^{\prime} \rightarrow 3^{\prime}\right)$ \\
\hline Ripk3-402 & GGUGAAGGCUAUGGUUAAUTT \\
Ripk3-1128 & CUCCGAGGUAAAGCAUUAUTT \\
Ripk3-493 & GCUCUGGUGACAAGAUUCATT \\
Ripk3-272 & GCCGUGAAGAACUGAAGAATT \\
Negative Control & UUCUCCGAACGUGUCACGUTT \\
GAPDH & CACUCAAGAUUGUCAGCAATT \\
\hline
\end{tabular}

胞分选. 利用流式细胞分选挑选GFP阳性的巨噬细胞, 获得稳转株. 定期观察检测巨噬细胞的GFP荧光情况. 定期复测转染效率.

将获得的GFP阳性的巨噬细胞进行分组，按照以 下3组进行刺激: A组 $2 \mu \mathrm{L}$ LPS $(100 \mathrm{ng} / \mathrm{mL})+200 \mu \mathrm{L}$ PBS; B组 $2 \mu \mathrm{L} \mathrm{LPS}(100 \mathrm{ng} / \mathrm{mL})+200 \mu \mathrm{L}$ 钛颗粒溶液; C 组 $2 \mu \mathrm{L} \mathrm{LPS}(100 \mathrm{ng} / \mathrm{mL})+200 \mu \mathrm{L} \operatorname{ATP}(10 \mathrm{mg} / \mathrm{mL})$. 分别 提取 3 组刺激后细胞的蛋白及细胞上清液中的分泌 蛋白.

\subsection{Real-time PCR, Western blot, ELISA}

Real-time PCR: 提取“方法1.4-刺激方法1”中不同 时间点的RNA样品. 检测目的基因为: RIPK3, NLRP3 以及 $I L-1 \beta$. 引物及内参的 PCR序列如表 2 所示. 将提取 好的RNA样品稀释至 $100 \mathrm{ng} / \mu \mathrm{L}$, 构建逆转录体系: $10 \mu \mathrm{L}$ 样品, $4 \mu \mathrm{L}$ 逆转录试剂Master-MiX, $6 \mu \mathrm{L}$ DEPC 水、震荡混匀. 程序设置: ( i ) $37^{\circ} \mathrm{C}, 15 \mathrm{~min}$; (ii) $85^{\circ} \mathrm{C}$, $5 \mathrm{~s}$; (iii) $4^{\circ} \mathrm{C}$ 保持, 将体系置于PCR仪, 开启上述程序, 获得complementary DNA(cDNA), 保存于 $-20^{\circ} \mathrm{C}$. 构建 Real-time PCR上样体系: $7 \mu \mathrm{L}$ DEPC水; $10 \mu \mathrm{L}$ SYBR Premix Ex Taq II; $2 \mu \mathrm{L}$ cDNA; $1 \mu \mathrm{L}$ 引物, 震荡混匀. 设 置2个副孔. 上机进行基因扩增. 使用2步法扩增: (i) $94^{\circ} \mathrm{C}, 30 \mathrm{~s}$; (ii) 扩增 $95^{\circ} \mathrm{C}, 5 \mathrm{~s}, 60^{\circ} \mathrm{C}, 20 \mathrm{~s}$, 一共进行 40 个循环. 数据分析: 使用LC480系统进行分析.

Western Blot：提取“方法1.4刺激方法2”中的蛋白 样本及方法 1.5 中的蛋白样本. 检测RIPK3蛋白(兔抗鼠 RIPK3单克隆抗体; CST, 美国)、NLRP3蛋白(兔抗鼠 NLRP3单克隆抗体; CST)、ASC蛋白(兔抗鼠ASC单克 隆抗体; Thermo Fisher Scientific, 美国)、Caspase-1蛋 白(兔抗鼠Caspase-1单克隆抗体; Abcam, 美国)及p20 蛋白水平(兔抗鼠p20单克隆抗体). 将提取好的蛋白标 
表 2 目的基因的引物序列

Table 2 Primer sequences of the target genes

\begin{tabular}{cc}
\hline PCR引物 & 引物序列 $\left(5^{\prime} \rightarrow 3^{\prime}\right)$ \\
\hline b-tublin-F & GCGCCTTTGGACACCTATTCA \\
b-tublin-R & GCCCTCCGTATAGTGCCCT \\
IL-1 $\beta-F$ & GAAATGCCACCTTTTGACAGTG \\
IL-1 $\beta-R$ & TGGATGCTCTCATCAGGACAG \\
NLRP3-F & ATTACCCGCCCGAGAAAGG \\
NLRP3-R & CATGAGTGTGGCTAGATCCAAG \\
RIPK3-F & ATCAACAGGCGAGACCTCTG \\
RIPK3-R & GTCCTCCTGGCATACCATAGA \\
GAPDH-F & TGACCTCAACTACATGGTCTACA \\
GAPDH-R & CTTCCCATTCTCGGCCTTG \\
\hline
\end{tabular}

本进行煮沸, 加入loading buffer并混匀. 按照实验规划 在SDS-聚丙烯酸胺凝胶孔内加入相同蛋白总量不同 体积蛋白样品. 电泳仪恒压 $80 \mathrm{~V}$, 保持 $30 \mathrm{~min}$, 待目的 条带跑到浓缩胶及分离胶分界线后, 改为恒压 $120 \mathrm{~V}$ 继 续电泳, 直到目的条带完全分开. 转膜后将转膜体系放 置于电转装置中，恒定电流 $220 \mathrm{~mA}$ 转膜 $2.5 \mathrm{~h}$. 取出条 带放置脱脂奶粉封闭 $1 \mathrm{~h}$ 后置于一抗及其稀释液中, $4{ }^{\circ} \mathrm{C}$ 下摇床孵育 $12 \mathrm{~h}$. 取出条带, 孵育二抗 $1 \mathrm{~h}$ 后使用智 能凝胶成像分析系统进行适度曝光. 保存原始图片.

ELISA：按照提取方法1.5中的细胞上清液中的分 泌蛋白. 使用ELISA试剂盒检测(Thermo Fisher Scientific)IL-1 $\beta$ 蛋白水平. 使用使用试剂盒中的标准蛋白试 剂构建标准曲线. 在ELISA试剂盒中加入细胞上清液. 根据试剂盒使用标准，反复加入试剂反应、孵育、加 入洗涤剂洗涤3次. 加入底物显色液 $100 \mathrm{~mL}$, 室温孵 育. 加入终止液. 根据蛋白标准曲线计算蛋白浓度.

\section{7 动物模型构建及分组}

本研究所有实验流程均严格遵守中山大学实验动 物福利伦理要求及规范使用实验室使用指南，并通过 中山大学肿瘤防治中心实验研究部动物实验中心动物 实验伦理批件(伦理号：L10202018060E). 本研究动物 来源于中山大学动物实验中心, 共购置24只8周龄specific pathogen free(SPF)级成年C57/BL6J雄性小鼠.

本课题共采用 24 只小鼠进行动物模型构建. 其中 24 只小鼠共分为 4 组, 每组 6 只小鼠. 具体分组及实验
方法如下: ( i ) Ti+PBS: 溶解有 $0.3 \mathrm{mg}$ 钛颗粒的 $30 \mu \mathrm{L}$ PBS $+70 \mu \mathrm{L}$ PBS; (ii) Ti+RIPK3-LV: 溶解有 $0.3 \mathrm{mg}$ 钛 颗粒的 $30 \mu \mathrm{L}$ PBS $+70 \mu \mathrm{L}$ 包含靶向抑制RIPK3-shRNA

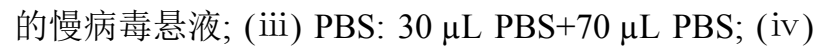
Sham: 假手术组.

采用腹腔注射 $1 \%$ 龙巴比妥钠 $(50 \mathrm{mg} / \mathrm{kg})$ 麻醉小鼠 后，以炒骨正中齐耳缘水平为中心作一长约 $1 \mathrm{~cm}$ 的纵 行切口, 充分暴露颖骨. 确定妙骨中缝区域, 使用神经 剥离子轻轻刮除颅骨骨膜5 10次，确保领骨骨膜完整 刮除. 裁剪一大小约为 $0.25 \mathrm{~cm} \times 0.25 \mathrm{~cm} \times 0.25 \mathrm{~cm}$ 明胶 海绵, 正中放置于小鼠颅骨颅缝区域.

按照分组依次在不同组别小鼠的颅骨内注入溶解 有 $0.3 \mathrm{mg}$ 钛颗粒的 $30 \mu \mathrm{L} \mathrm{PBS}+70 \mu \mathrm{L}$ PBS; 溶解有 $0.3 \mathrm{mg}$ 钛颗粒的 $30 \mu \mathrm{L} \quad \mathrm{PBS}+70 \mu \mathrm{L}$ 包含靶向抑制 RIPK3-shRNA的慢病毒悬液; $30 \mu \mathrm{L}$ PBS $+70 \mu \mathrm{L}$ PBS. 提起小鼠头乑皮肤, 使悬液不溢出. 使用可吸收缝线缝 合切口. 2周后用颈椎脱臼法处死小鼠并获取小鼠㐿骨 标本, 将小鼠颖骨周围组织剔除干净后进行 micro-CT 扫描, 并对图像进行三维重建及测量.

\section{8 小鼠颖骨TRAP染色}

小鼠颖骨标本进行脱钙处理：将小鼠㐿骨浸泡于 $10 \%$ 的脱钙液 30 天脱钙，将脱钙后的小鼠颖骨进行石 蜡包埋, 分别切成 $3 \mu \mathrm{m}$ 厚的切片, 分别进行HE(hematoxylin-eosin)和TRAP(tartrate resistant acid phosphatase)染色. 使用于10X视野下观察切片. 定位至小鼠颖 缝处, 可观察到TRAP染色切片中的破骨细胞. 破骨细 胞胞浆为深红色, 细胞周围有骨吸收陷窝, 内含有深紫 色颗粒. 统计小鼠颅骨切片中的破骨细胞数, 取平均 值. 独立样本 $t$ 检验进行组间比较.

\section{2 实验结果}

2.1 无菌性松动组关节周围软组织中NLRP3, RIPK3，IL-1及的蛋白水平表达明显高于股骨头坏死组

无菌性松动组患者的髋关节周围软组织的HE染 色标本中可见大量黑色致密金属沉积(图1). 免疫组化 结果可发现，股骨头坏死组患者的髋关节周围滑膜组 织与无菌性松动组患者的髋关节周围组织均存在IL$1 \beta$ 大量表达, 但无菌性松动组患者的IL- $1 \beta$ 表达量明显 多于股骨头坏死组患者(图2A)，股骨头坏死组患者的 


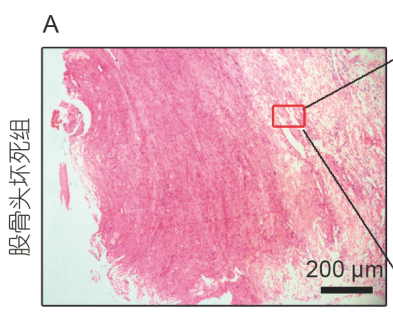

B
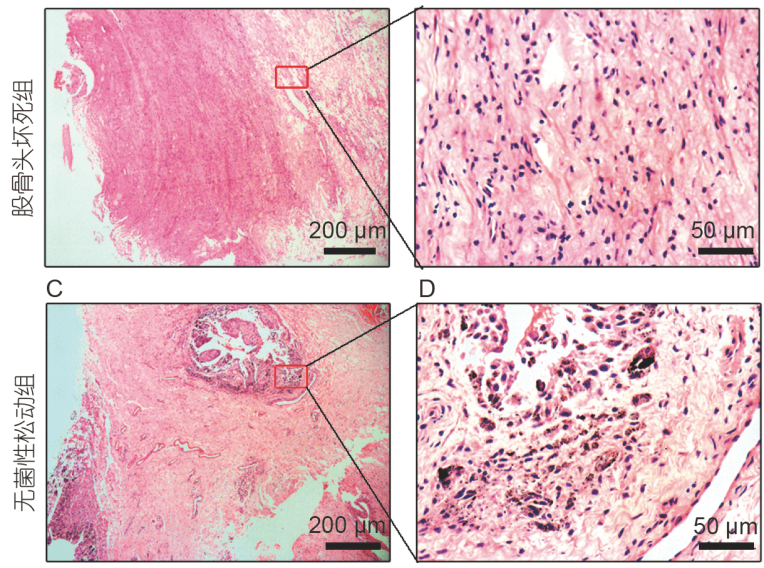

图 1 股骨头坏死组及无菌性松动组关节周围软组织HE染 色. C和D分别为 $\mathrm{A}$ 和 $\mathrm{B}$ 中红色方框所选定区域

Figure 1 HE staining of soft tissue around the joints in the femoral head necrosis group and the aseptic loosening group. $\mathrm{C}$ and $\mathrm{D}$ are the areas selected by the red boxes in A and B, respectively

髋关节周围滑膜组织中RIPK3, NLRP3无明显表达, 而 在无菌性松动组患者的髋关节周围组织中RIPK3, NLRP3蛋白大量表达(图2A). Bresaliers染色半定量分 析发现(图2B), 无菌性松动组患者髋关节周围组织中 IL-1 $\beta$ 的评分为 $2.83 \pm 0.17$, 显著高于股骨头坏死组患者 $1.67 \pm 0.13(P<0.05)$, 说明无菌性松动患者的髂关节周 围组织中促炎因子高度表达. 同时发现，RIPK3及
NLRP3也在无菌性松动组患者中表达增强：无菌性松 动组患者髋关节周围组织中 RIPK 3 的染色评分为 1.82 \pm 0.16 ，显著高于股骨头坏死组染色评分 $0.18 \pm 0.08$ $(P<0.05)$; NLRP3的染色评分为 $1.45 \pm 0.21$, 高于股骨头 坏死组染色评分 $0.16 \pm 0.12(P<0.05)$.

\section{2 磨损颗粒刺激小鼠巨噬细胞后, IL-1ß, RIPK3 及NLRP3表达上调, 且p20的表达量也同步上调}

使用磨损颗粒刺激巨噬细胞J774A.1后, 各时间点 $(1 \mathrm{~h}, 2 \mathrm{~h}, 4 \mathrm{~h}, 6 \mathrm{~h})$ 的IL-1 1 的mRNA表达量均明显上调 (图3C), 在此过程中RIPK3, NLRP3的mRNA表达量也 同步上调(图3A和B). 磨损颗粒刺激巨噬细胞J774A.1 后检测NLRP3蛋白通路可发现，NLRP3蛋白表达量和 p20蛋白表达量均明显上调(图3D), 表明磨损颗粒刺激 不仅能上调NLRP3蛋白表达，同时能促进NLRP3炎性 小体的组装.

\section{3 靶向敲低RIPK3 基因后, 磨损颗粒刺激巨噬细 胞产生的IL-1及表达量均明显下调}

验证 $R I P K 3$ 基因敲低后，用钛颗粒刺激慢病毒转 染的巨噬细胞J774A.1, 与刺激后的 NC(negative control)转染巨喍细胞相比, IL-1 $\beta$ 分泌蛋白表达显著降低 (图4). 在阳性对照组(LPS+ATP刺激组)也观察到类似
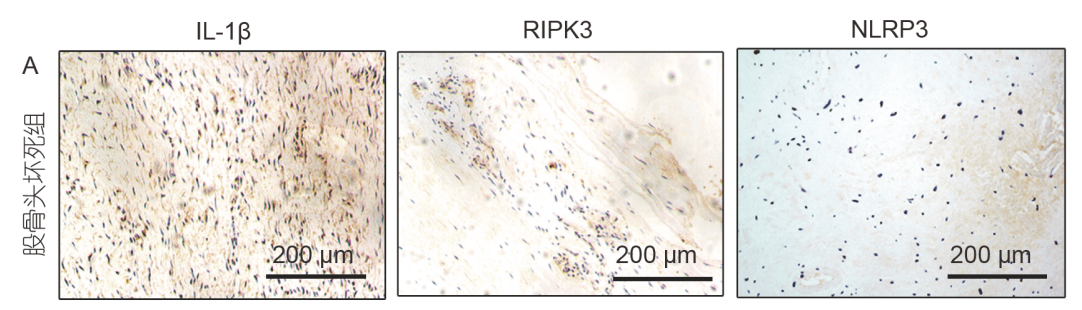

B
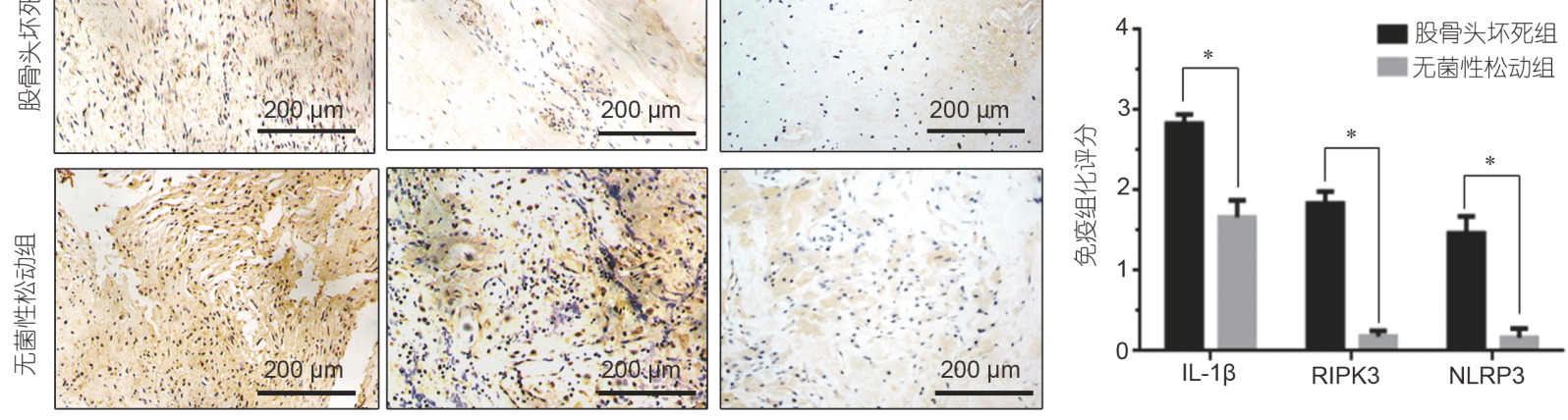

图 2 股骨头坏死组及无菌性松动组关节周围软组织免疫组化染色及半定量Bresaliers染色评分. A: 股骨头坏死组及无菌性 松动组关节周围软组织中IL-1 $\beta$, RIPK3, NLRP3蛋白免疫组化染色, 棕色代表染色蛋白; B: 股骨头坏死组及无菌性松动组关 节软组织中IL-1 $\beta$, RIPK3, NLRP3的半定量染色评分, 其中*表示同组内比较 $P<0.05$

Figure 2 Immunohistochemical staining and semi-quantitative Bresaliers staining of soft tissue around the joints in the femoral head necrosis group and the aseptic loosening group. A: Immunohistochemical staining of IL-1 $\beta$, RIPK3, and NLRP3 proteins in soft tissue around the joints of the femoral head necrosis group and the aseptic loosening group. Brown represents stained protein; B: semi-quantitative staining scores of IL-1 $\beta$, RIPK3, and NLRP3 in articular soft tissue of the femoral head necrosis group and the aseptic loosening group. * indicates the comparison within the same group was statistically significant, $P<0.05$ 


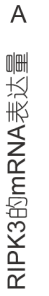
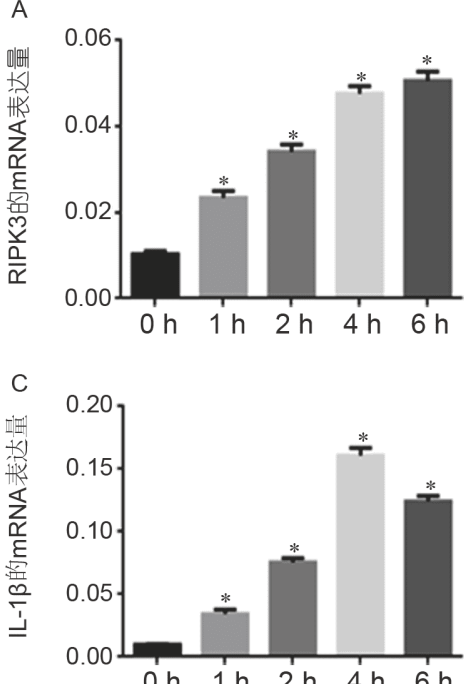

B

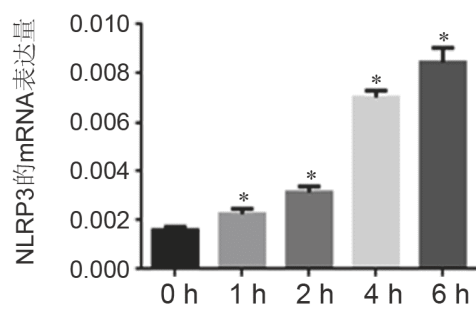

D

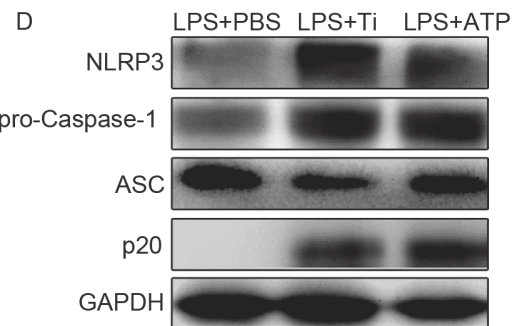

图 3 磨损颗粒刺激巨噬细胞后IL-1 $\beta$, RIPK3, NLRP3及NLRP3信号通路的表达改变. A C: PCR示钛颗粒刺激巨噬细胞后各 个时间点RIPK3, NLRP3, IL-1 $\beta$ 的mRNA表达显著增高 $(*$ 表示和 $0 \mathrm{~h}$ 相比具有明显差异, $P<0.05)$; D: Western Blot显示LPS+PBS, LPS+Ti, LPS+ATP刺激下巨噬细胞的NLRP3通路蛋白表达量, 其中NLRP3蛋白及p20蛋白的表达量明显升高

Figure 3 Changes in the expression of IL-1 $\beta$, RIPK3, NLRP3, and NLRP3 signaling pathways after macrophages are stimulated by abrasion particles. A-C: PCR shows that mRNA expression of RIPK3, NLRP3, and IL-1 $\beta$ increased significantly at various time points after titanium particles stimulated the macrophages (* indicates a significant difference compared with the mRNA expression at $0 \mathrm{~h}, P<0.05$ ); D: the expression of NLRP3 pathway protein in macrophages was stimulated by LPS+PBS, LPS+Ti, LPS+ATP, and the expression of NLRP3 protein and p20 protein were significantly increased

的结果，转染RIPK3慢病毒的巨噬细胞释放IL-1 $\beta$ 的表 达量明显降低. 说明转染靶向抑制RIPK3慢病毒的巨 噬细胞在磨损颗粒激活产生的促炎作用明显减弱.

2.4 靶向敲低 $R I P K 3$ 基因后, 颗粒刺激的巨噬细胞 中p20表达量明显降低，表明颗粒刺激巨噬细胞中 的NLRP3炎性小体组装受限

Western Blot检测慢病毒转染后巨噬细胞的 NLRP3信号通路改变情况，钛颗粒刺激下慢病毒转染 后巨噬细胞的NLRP3蛋白明显升高，但与钛颗粒刺激 后的NC转染细胞相比无明显差异(图5). 说明RIPK3在 磨损颗粒激活巨噬细胞的过程中不影响NLRP3蛋白 的表达. 但钛颗粒刺激后巨噬细胞中的 $\mathrm{p} 20$ 表达量与 $\mathrm{NC}$ 转染巨噬细胞相比表达量明显降低(图5), 说明 NLRP3炎性小体的组装受到限制。以上结果提示, RIPK3 在磨损颗粒激活巨噬细胞的过程中可能参与调 控NLRP3炎性小体的组装.

\section{5 注射靶向抑制RIPK3 慢病毒可明显缓解磨损 颗粒诱导的骨溶解效应}

注射靶向抑制RIPK3慢病毒的小鼠㐿骨与单纯注

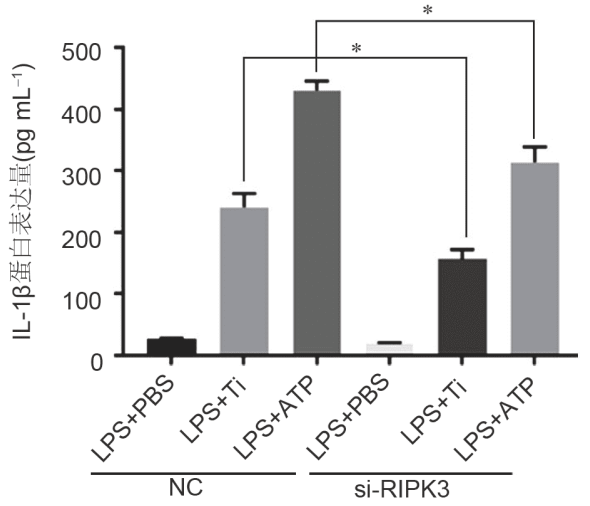

图 4 ELISA检测LPS+PBS, LPS+Ti，LPS+ATP刺激下 RIPK3 慢病毒转染巨喍细胞和NC巨噬细胞的IL-1 $\beta$ 分泌量 $(*$, $P<0.05)$

Figure 4 Detection of IL-1 $\beta$ secretion of RIPK3 lentivirus-transfected macrophages and NC macrophages under LPS+PBS, LPS+Ti, and LPS + ATP stimulation by ELISA $(*, P<0.05)$

射钛颗粒的小鼠颅骨在预骨领缝区都出现不同程度的 骨溶解表现(图6)，但注射靶向抑制RIPK3慢病毒的小 鼠颅骨溶骨效应远远弱于单纯磨损钛颗粒刺激的小鼠 颅骨溶骨效应. 定量分析提示, 注射靶向抑制RIPK3慢 病毒后，小鼠炒缝局部BV/TV(bone volume/total volume $)$ 提高了 $61 \%(P<0.05)$, 骨密度提高了 $56.1 \%$ 


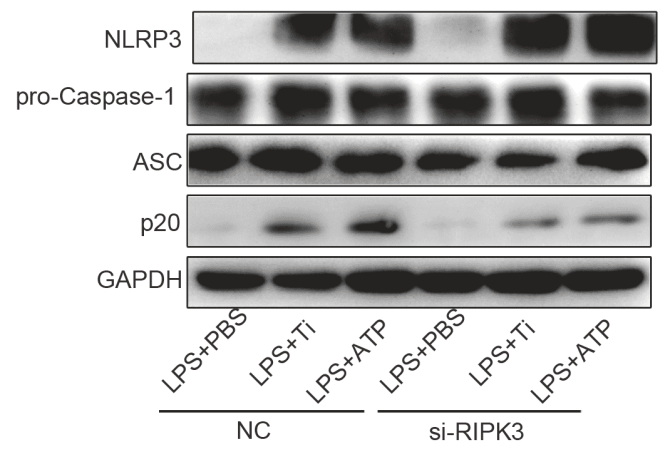

图 5 Western blot检测LPS+PBS, LPS+Ti, LPS+ATP刺激下 慢病毒转染巨噬细胞和NC转染巨噬细胞的NLRP3信号通路 改变. RIPK3慢病毒转染细胞的p20表达量与NC转染巨噬细 胞相比表达量明显降低

Figure 5 Western blot detection of NLRP3 signaling pathway changes in lentiviral transfected macrophages and $\mathrm{NC}$ transfected macrophages stimulated by LPS+PBS, LPS+Ti, and LPS+ATP. The expression of p20 in RIPK3 lentivirus-transfected cells was significantly lower than that in NC-transfected macrophages

\section{$(P<0.05)$ (图6). 转染靶向抑制RIPK3慢病毒可有效缓解} 小鼠乑骨溶解模型的骨溶解效应.

小鼠频骨的病理切片可见，注射靶向抑制RIPK3 慢病毒的小鼠颅骨及单纯注射钛颗粒的小鼠颅骨在硕

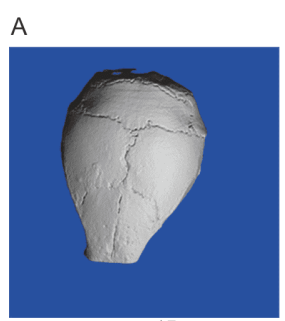

PBS组

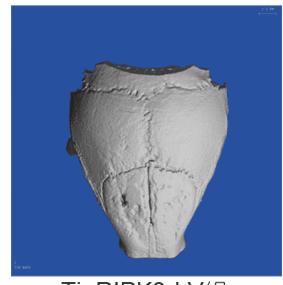

Ti+RIPK3-LV组

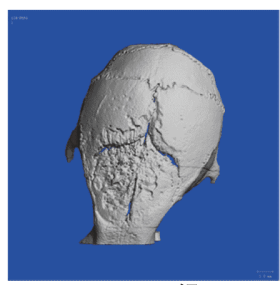

Ti+PBS 组

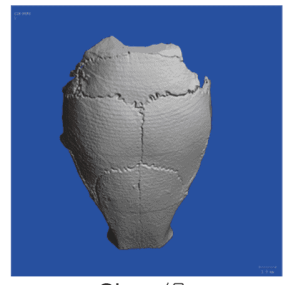

Sham组
骨颅缝区都有不同程度的破骨细胞聚集(图7A)。在硕 骨颎缝中心计算视野下破骨细胞数, 结果提示, $\mathrm{Ti}$ + PBS组和Ti+RIPK3-LV组颅缝周围的破骨细胞数之 间没有统计学差异 $(P>0.05)$ (图7B).

\section{3 讨论}

人工关节置换术后关节假体松动是目前骨科界所 面临的最严峻的难题之一 ${ }^{[19]}$. 关节松动的患者通常要 面临翻修的风险. 综合全球的调查研究也表明, 无菌 性松动是发生假体松动的最主要原因. 目前普遍认为, 磨损颗粒诱导的假体周围骨溶解是无菌性松动发生的 主要机制. 通常认为, 磨损颗粒激活巨噬细胞的PRRs 释放促炎因子是引起人工关节无菌性松动的最主要原 因. 本课题组既往研究也表明, 磨损颗粒本身(不含内 毒素)可通过经典激活方式刺激巨噬细胞产生促炎因

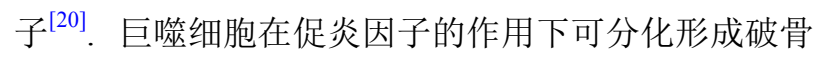
细胞, 参与假体周围骨溶解破坏 ${ }^{[21]}$.

本课题组通过体内外实验研究表明，RIPK3在磨 损颗粒激活巨噬细胞PRRs/NLRP3信号通路引起炎症
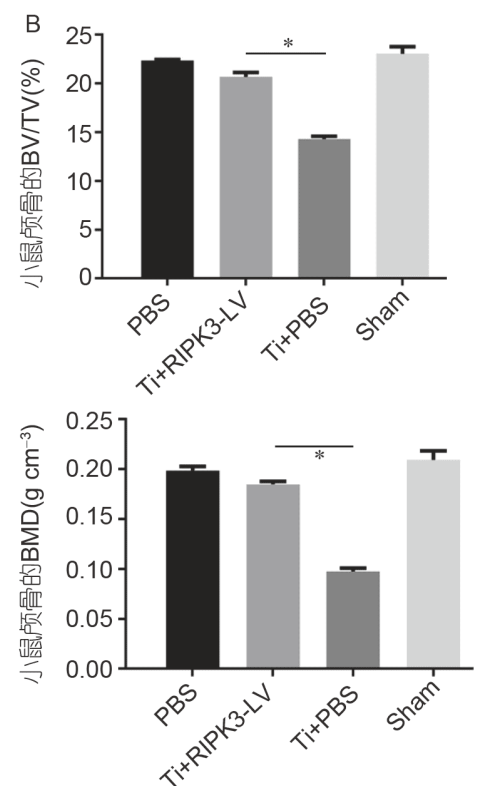

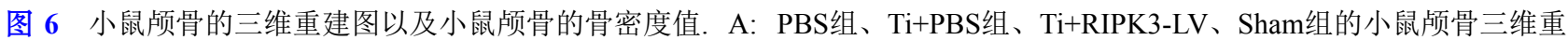
建图, 可直观观察到Ti-PBS组的骨溶解效应最强; B: PBS组、Ti+PBS组、Ti+RIPK3-LV、Sham组的小鼠㐿缝去的BV/TV值及 骨密度值. * $P<0.05$

Figure 6 The three-dimensional reconstruction and the bone mineral density (BMD) of the mouse skull. A: Three-dimensional reconstruction of the skull of mice in the PBS group, Ti+PBS group, Ti+RIPK3-LV, and Sham group. It can be directly observed that the osteolysis effect of the Ti-PBS group is the strongest. B: BV/TV and BMD values of cranial sutures of mice in the PBS group, Ti+PBS group, Ti+RIPK3-LV, and Sham group. *, $P<0.05$ 


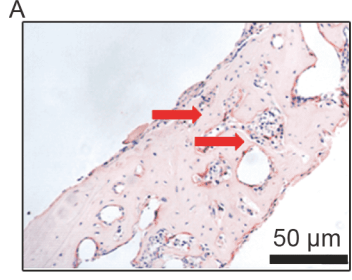

PBS组

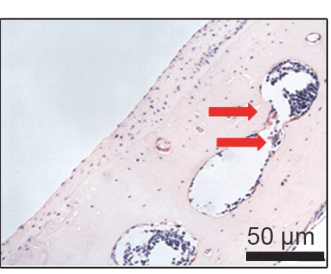

Ti+RIPK3-LV组

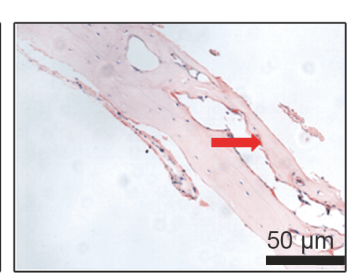

Ti+PBS 组

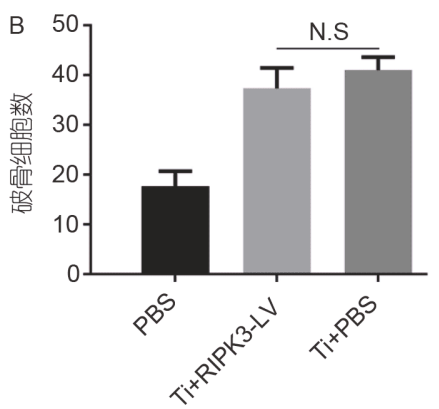

图 7 小鼠颅缝TRAP染色及破骨细胞计数. A: PBS组、 Ti+PBS组、Ti+RIPK3-LV的小鼠颖缝TRAP染色, 箭头所指细胞为破 骨细胞; B: PBS组、Ti+PBS组、Ti+RIPK3-LV的小鼠颅缝TRAP细胞数统计比较 $(\mathrm{N} . \mathrm{S}$.表示 $P>0.05)$

Figure 7 TRAP staining and osteoclast number in mouse craniotomy. A: TRAP staining of mouse skull in PBS group, Ti+PBS group, and Ti + RIPK3-LV. The cells indicated by the arrows are osteoclasts. B: Statistical comparison of the number of TRAP cells in the skull of mice in the PBS group, Ti+PBS group, and Ti+RIPK3-LV (N.S. means $P>0.05$ )

反应中起正性调控作用. 本课题组发现, 单纯钛颗粒刺 激巨噬细胞J774A.1后，巨噬细胞中的RIPK3及NLRP3 的mRNA及蛋白表达量均明显升高. 既往研究发现, 巨 噬细胞中NF- $\kappa$ B 通路的激活可上调NLRP3 mRNA的表 达，后者再进一步进行翻译和翻译后修饰，从而上调 NLRP3蛋白表达, 这个步骤也称为NLRP3的priming $^{[22]}$. 在静息状态下, NLRP3的表达量几乎可以忽略 不计, 只有在NF- $\mathrm{kB}$ 激活时, NLRP3才会进行转录及翻 译. 通常认为, 参与调控巨噬细胞NF- $\kappa B$ 通路的分子也 能影响NLRP3的priming阶段 ${ }^{[23]}$. 本课题组认为, 钛颗 粒上调NLRP3的蛋白表达水平的分子机制可能和磨 损颗粒经典激活巨噬细胞机制相关. 既往研究表明, 单 核/巨筮细胞具有两种不同的激活途径 ${ }^{[24]}$ : 第一种是经 典激活途径, 主要由脂多糖激活, 有时也可由 I 型辅助 T淋巴细胞因子( $\mathrm{T}$ helper 1 cell, Th1)激活, 主要表达 TNF- $\alpha$, MMP-7等促炎因子. 此类激活方式多与急性炎 症的发生相关, 所产生的促炎因子有组织破坏作用. 第 二种激活方式是选择性激活途径，巨噬细胞在供氧不 足的环境中表达IL-10, PDGF等细胞因子而非促炎因 子，这些细胞因子在机体损伤状态下能参与机体的功 能修复. 本课题组 ${ }^{[25]}$ 前期研究发现, 磨损颗粒可以通 过识别巨噬细胞TLRs，激活其下游的NF- $\mathrm{kB}$ 通路，产 生促炎因子，通过经典激活途径激活巨噬细胞. 由此 本课题组认为, 钛颗粒激活NLRP3启动priming过程可 能与其识别TLR受体激活NF- $\mathrm{KB}$ 通路的方式有关.

NLRP3蛋白大量表达后能与ASC, pro-Caspase-1 组装形成NLRP3炎性小体, NLRP3炎性小体切割proCaspase-1形成活性Caspase-1(p20), 后者切割pro-IL-
$1 \beta$, pro-IL-18形成活性促炎因子并释放至细胞外，从 而发挥促炎作用 ${ }^{[26]}$. 活性Caspase-1 是NLRP3炎性小体 形成并发挥促炎作用的标志，因此通常检测巨噬细胞 中p20的表达量可验证NLRP3炎性小体是否组装完 成 ${ }^{[27]}$. NLRP3发挥促炎作用一般需要两个上游信号激 活，第一信号促进NLRP3启动priming过程，NLRP3蛋 白大量表达; 第二信号启动NLRP3蛋白与其他配体组 装形成炎性小体, 从而发挥促炎作用 ${ }^{[28,29]}$. 在本课题 中, 单纯钛颗粒刺激后NLRP3的mRNA表达升高, 说 明磨损钛颗粒可作为第一信号激活NLRP3的priming 过程. 同时单纯钛颗粒刺激后IL-1 $1 \beta$ 的表达量上调, 使 用钛颗粒混合LPS刺激巨噬细胞后, NLRP3蛋白、IL$1 \beta 、 \mathrm{p} 20$ 的表达量均明显上调, 显示钛颗粒可以激活 NLRP3的组装过程, 促进NLRP3发挥促炎作用. 在本 课题中, ELISA检测结果显示, 单纯钛颗粒刺激巨噬细 胞后产生促炎因子的表达量有所上调, 但远小于钛颗 粒混合LPS共同刺激巨噬细胞所产生的促炎因子蛋白 表达量. 既往也有文献在体外实验中证明, 磨损颗粒作 为第一信号的刺激作用较弱，作为第二信号促进 NLRP3炎性小体组装的作用较强 ${ }^{[30]}$.

本课题选用的体内模型是小鼠炒骨溶解模型, 这 是目前为止较为成熟的颗粒介导的无菌性松动动物模 型. 无菌性松动的动物模型较难构建, 通常认为, 无菌 性松动的过程难以模拟, 需要长期复杂的生理反应, 是 磨损颗粒及机体组织发生相互物理、生化作用 ${ }^{[31]}$. 合 格的无菌性松动的动物模型的构建应该具有以下 3 点 特点: (i) 在组织学上能模拟无菌性松动的生理过程; (ii) 在生物力学上能体现人工关节假体的应力方式; 
(iii) 为探讨磨损颗粒引起人工关节无菌性松动提供治 疗靶点.

在小鼠颖骨表面注入靶向抑制RIPK3慢病毒后， 小鼠颓骨溶解效应明显减弱. 这为靶向抑制RIPK3治 疗无菌性松动的发生提供了重要的可能性. 目前为止 尚无RIPK3与骨质疏松或骨关节疾病等相关疾病相联 系的文献报道，本课题首次将RIPK3与人工关节无菌 性松动相联系. 然而, 既往研究发现RIPK3在心血管、 皮肤等相关疾病中具有明确的作用. 通常认为, RIPK3 参与各种急性和慢性炎症反应，在机体自身的免疫反 应和炎症反应中都起关键性作用。在心血管疾病中, RIPK3 和心肌的缺血再灌注以及动脉粥样硬化密切相 关. 当动脉粥样硬化进展至不可逆期时, 心血管巨噬细 胞中的RIPK3会启动程序性坏死，造成不可逆的心肌 细胞炎症性破坏，同时炎症因子释放的增多会正反馈 激活RIPK3 表达, 从而造成进一步的破坏和损伤 ${ }^{[32]}$. 另 有研究发现，NLRP3炎性小体也参与心肌缺血再灌注 的病理过程. 缺血再灌注损伤时，血管内皮细胞中硫 氧还蛋白能促进激活NLRP3炎性小体，从而加剧心肌 细胞损伤 ${ }^{[33]}$. 但在此过程中的具体炎症机制尚不明确.

本实验表明, 敲低RIPK3后, NLRP3炎性小体的组 装受限，磨损颗粒的促炎作用减弱. 近年来RIPK3对 NLRP3炎性小体的调控机制研究十分热门，但具体的 调控方式一直存在争议. 通常认为, RIPK3参与组织细 胞的炎性体依赖性细胞死亡，也称为pyroptosis ${ }^{[34]}$. 炎 性体依赖性细胞死亡的特征在于细胞内组装形成各种 炎性小体, 加工和释放IL- $1 \beta$, 导致细胞进入炎性状态. Yabal等人 ${ }^{[35]}$ 研究发现, 当IAP(inhibitor of apoptosis protein)受到抑制时，BMDM(bone marrow derived macrophage)中的RIPK3 能激活NLRP3炎性小体的组 装，同时IL-1 $\beta$ 的蛋白表达量也会上调. 另有文献表 明 $^{[36]}$, RIPK3 调控NLRP3炎性小体的活性多涉及2种不 同的信号转导方式,一种涉及坏死效应蛋白MLKL, 促 进细胞调亡. 另一种涉及线粒体裂变蛋白DRP1, 主要 与炎症效应的产生有关. 线粒体裂变时会产生和释放 mtROS(mitochondrial reactive oxygen), 释放的mtROS 能通过NLRP3信号通路引起炎症反应 ${ }^{[37]}$. 在本课题中, 线粒体调亡与NLRP3炎性小体组装受限之间是否有 必然的联系, 目前尚不明确.

\section{4 结论}

RIPK3 在磨损颗粒激活巨噬细胞PRRs/NLRP3信 号通路引起炎症反应中起正性调控作用. 在小鼠㐿骨 溶解模型中，局部注射靶向抑制RIPK3 慢病毒能有效 抑制小鼠杪骨溶解模型的骨溶解效应，抑制效率约为 $60 \%$. RIPK3 在磨损颗粒诱导的小鼠颖骨溶解模型中 起正向调控作用.

\section{参考文献}

1 Shan L, Shan B, Graham D, et al. Total hip replacement: A systematic review and meta-analysis on mid-term quality of life. Osteoarthritis Cartilage, 2014, 22: 389-406

2 Higuchi Y, Hasegawa Y, Seki T, et al. Significantly lower wear of ceramic-on-ceramic bearings than metal-on-highly cross-linked polyethylene bearings: A 10- to 14-year follow-up study. J Arthroplasty, 2016, 31: 1246-1250

3 Prieto-Alhambra D, Javaid M K, Judge A, et al. Association between bisphosphonate use and implant survival after primary total arthroplasty of the knee or hip: population based retrospective cohort study. BMJ, 2011, 343: d7222

4 Sundfeldt M, Carlsson L V, Johansson C B, et al. Aseptic loosening, not only a question of wear: A review of different theories. Acta Orthop, 2006, 77: 177-197

5 Ding $\mathrm{Y}$, Qin $\mathrm{C}, \mathrm{Fu} \mathrm{Y}$, et al. In vitro comparison of the biological activity of alumina ceramic and titanium particles associated with aseptic loosening. Biomed Mater, 2012, 7: 045019

6 Mukherjee S, Karmakar S, Babu S P S. TLR2 and TLR4 mediated host immune responses in major infectious diseases: A review. Braz J Infect Dis, 2016, 20: 193-204

7 Zhang S, Weinberg S, DeBerge M, et al. Efferocytosis fuels requirements of fatty acid oxidation and the electron transport chain to polarize macrophages for tissue repair. Cell Metab, 2019, 29: 443-456.e5

8 Liu Q, Ding J L. The molecular mechanisms of TLR-signaling cooperation in cytokine regulation. Immunol Cell Biol, 2016, 94: 538-542 
9 Liu B Y, Cai B S, Gao C J. Regulation of innate antiviral immunity by protein ubiquitination (in Chinese). Sci Sin Vitae, 2018, 48: 1152-1161 [刘 冰玉, 蔡宝珊, 高成江. 泛素化调控抗病毒天然免疫的研究进展. 中国科学: 生命科学, 2018, 48: 1152-1161]

$10 \mathrm{Xu} \mathrm{C}$, Lu Z, Luo Y, et al. Targeting of NLRP3 inflammasome with gene editing for the amelioration of inflammatory diseases. Nat Commun, 2018, 9: 4092

11 Qing $\mathrm{L}, \mathrm{Fu}$ J, Wu P, et al. Metformin induces the M2 macrophage polarization to accelerate the wound healing via regulating AMPK/mTOR/ NLRP3 inflammasome singling pathway. Am J Transl Res, 2019, 11: 655-668

12 Moriwaki K, Chan F K M. RIP3: A molecular switch for necrosis and inflammation. Genes Dev, 2013, 27: 1640-1649

13 Chow M T, Duret H, Andrews D M, et al. Type I NKT-cell-mediated TNF- $\alpha$ is a positive regulator of NLRP3 inflammasome priming. Eur J Immunol, 2014, 44: 2111-2120

14 Deng Q, Geng Y, Zhao L, et al. NLRP3 inflammasomes in macrophages drive colorectal cancer metastasis to the liver. Cancer Lett, 2019, 442: 21-30

15 Declercq W, Vanden Berghe T, Vandenabeele P. RIP kinases at the crossroads of cell death and survival. Cell, 2009, 138: 229-232

16 Wang X, Jiang W, Yan Y, et al. RNA viruses promote activation of the NLRP3 inflammasome through a RIP1-RIP3-DRP1 signaling pathway. Nat Immunol, 2014, 15: 1126-1133

17 Lawlor K E, Khan N, Mildenhall A, et al. RIPK3 promotes cell death and NLRP3 inflammasome activation in the absence of MLKL. Nat Commun, 2015, 6: 6282

18 Bresalier R S, Ho S B, Schoeppner H L, et al. Enhanced sialylation of mucin-associated carbohydrate structures in human colon cancer metastasis. Gastroenterology, 1996, 110: 1354-1367

19 Liang P, Niu S. Early diagnosis of artificial joint aseptic loosening after total joint arthroplasty (in Chinese). Chin J Joint Surg (Electron Ed), 2019,13: 99-104 [梁鹏, 牛舜. 人工关节置换术后假体无菌性松动的早期诊断. 中华关节外科杂志(电子版), 2019, 13: 99-104]

20 Qin C, Ding Y, Huang D, et al. Down-regulation of TNF-alpha by small interfering RNA inhibits particle-induced inflammation in vitro. Artif Organs, 2011, 35: 706-714

21 El Fiky A, Perreault R, McGinnis G J, et al. Attenuated expression of interferon- $\beta$ and interferon- $\lambda 1$ by human alternatively activated macrophages. Hum Immunol, 2013, 74: 1524-1530

22 Qiao Y, Wang P, Qi J, et al. TLR-induced NF-kB activation regulates NLRP3 expression in murine macrophages. FEBS Lett, 2012, 586: 10221026

23 Vande Walle L, Van Opdenbosch N, Jacques P, et al. Negative regulation of the NLRP3 inflammasome by A20 protects against arthritis. Nature, 2014, 512: 69-73

24 Medzhitov R, Preston-Hurlburt P, Janeway Jr C A. A human homologue of the Drosophila Toll protein signals activation of adaptive immunity. Nature, 1997, 388: 394-397

25 Zhang C, Li C, Li S, et al. Small heterodimer partner negatively regulates TLR4 signaling pathway of titanium particles-induced osteolysis in mice. J Biomed Nanotechnol, 2018, 14: 609-618

26 Qin Y, Zhao W. Negative regulatory mechanisms of NLRP3 inflammasome (in Chinese). Sci Sin Vitae, 2018, 48: 1187-1196 [秦颖, 赵伟. NLRP3炎症小体的负向调控机制. 中国科学: 生命科学, 2018, 48: 1187-1196]

27 Wang W, Mao S, Yu H, et al. Pinellia pedatisecta lectin exerts a proinflammatory activity correlated with ROS-MAPKs/NF- $\mathrm{kB}$ pathways and the NLRP3 inflammasome in RAW264.7 cells accompanied by cell pyroptosis. Int Immunopharmacol, 2019, 66: 1-12

28 Zhang Y, Yang X, Qiu C, et al. Matrine suppresses AGE-induced HAEC injury by inhibiting ROS-mediated NRLP3 inflammasome activation. Eur J Pharmacol, 2018, 822: 207-211

29 Dong N, Shao F. Molecular mechanism and immunological function of pyroptosis (in Chinese). Sci Sin Vitae, 2019, 49: 1606-1634 [董娜, 召峰. 细胞焦亡的机制和功能. 中国科学: 生命科学, 2019, 49: 1606-1634]

30 Manzano G W, Fort B P, Dubyak G R, et al. Wear particle-induced priming of the NLRP3 inflammasome depends on adherent pathogenassociated molecular patterns and their cognate Toll-like receptors: An in vitro study. Clin Orthop Relat Res, 2018, 476: 2442-2453

31 Cheng T, Zhao Y, Li B, et al. Curcumin attenuation of wear particle-induced osteolysis via RANKL signaling pathway suppression in mouse calvarial model. Mediators Inflamm, 2017, 2017: 1-11

32 Lin J, Li H, Yang M, et al. A role of RIP3-mediated macrophage necrosis in atherosclerosis development. Cell Rep, 2013, 3: 200-210

33 Liu C, Tang Q Z. Research progress of NLRP3 in cardiac remodeling (in Chinese). Chin J Geriatr Heart Brain Vesel Dis, 2019, 21: 1327-1329 
[刘晨, 唐其柱. 核苷酸结合寡聚化结构域样受体蛋白3在心肌重构中的研究进展. 中华老年心脑血管病杂志, 2019, 21: 1327-1329]

34 Croker B A, O'Donnell J A, Gerlic M. Pyroptotic death storms and cytopenia. Curr Opin Immunol, 2014, 26: 128-137

35 Yabal M, Müller N, Adler H, et al. XIAP restricts TNF- and RIP3-dependent cell death and inflammasome activation. Cell Rep, 2014, 7: 17961808

36 Kang T B, Yang S H, Toth B, et al. Caspase-8 blocks kinase RIPK3-mediated activation of the NLRP3 inflammasome. Immunity, 2013, 38: 2740

37 Chen L B, Ma K L, Chen Q, et al. Mechanism of mitophagy in cell homeostasis (in Chinese). Sci Sin Vitae, 2019, 49: 1045-1053 [陈林波, 马凯 丽, 陈佺, 等. 线粒体自噬的分子机制. 中国科学: 生命科学, 2019, 49: 1045-1053]

\title{
Mechanism of RIPK3 in activation of PRRs/NLRP3 signaling pathway in macrophages induced by wear particles in aseptic joint loosening
}

\author{
PENG Peng, FU Yuan, QIU JunXiong, LI ShiXun, LI ChangChuan, KUANG ManYuan, \\ FANG GuiBin, LIN SiPeng \& DING Yue \\ Department of Orthopaedics, Sun Yixian Memorial Hospital, Sun Yat-sen University, Guangzhou 510120, China
}

\begin{abstract}
Arthroplasty is the most effective method for treating end-stage bone and joint disease in the world. However, the artificial joint prosthesis has a limited service life. It is known that wear particles could stimulate macrophages to produce proinflammatory cytokine, which activates the osteoclasts around the prosthesis, and play the most important role in aseptic loosening. The particles can activate pattern recognition receptors (PRRs) of macrophages to produce pro-inflammatory factors such as TNF- $\alpha$ and IL-1 $\beta$, and induce osteolysis around the prosthesis. Based on previous research, we found that in mouse macrophages with the RIPK3 gene inhibited, the expression of proinflammatory factors induced by wear particles was significantly reduced, and the assembly of NLRP3 inflammatory bodies was limited. At the same time, it was found that the effects of cranium lysis were weakened in mice injected with lentivirus targeting to inhibit RIPK3. This study shows that RIPK3 plays a positive regulatory role in the inflammatory response caused by wear particles activating macrophage PRRs/NLRP3 signaling pathway.
\end{abstract}

RIPK3, NLRP3, artificial joint replacement, aseptic loosening, macrophages

doi: $10.1360 /$ SSV-2020-0016 